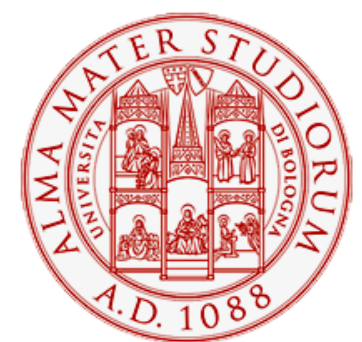

Alma Mater Studiorum - Università di Bologna DEPARTMENT OF ECONOMICS

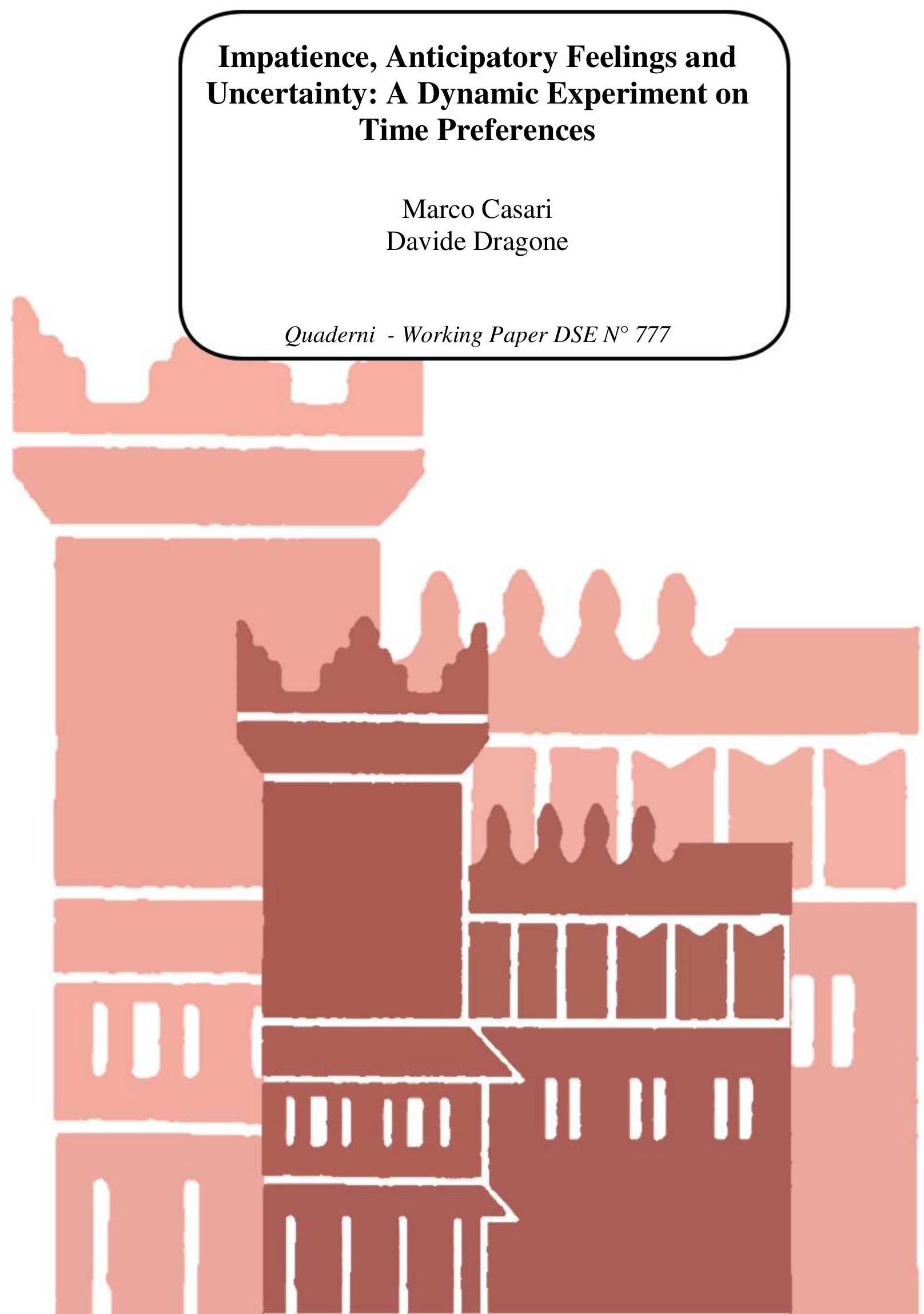




\title{
Impatience, Anticipatory Feelings and Uncertainty: A Dynamic Experiment on Time Preferences*
}

\author{
Marco Casari Davide Dragone
}

July 22, 2011

\begin{abstract}
We study intertemporal choices through an experiment that elicits a subject's plan and then tracks its implementation over time. There are two main results. When facing a costly task to be completed under a deadline, two thirds of subjects prefer anticipating it rather than postponing it. Choice reversals are common although present-biased preferences alone cannot explain them. This evidence is compatible with models based on anticipatory feelings and stochastic utility.
\end{abstract}

JEL codes: C91, D01, D80, D90

Keywords: time inconsistency, self-control, risk, stochastic utility, real-effort experiment

${ }^{*}$ Correspondence address: University of Bologna, Piazza Scaravilli 2, 40126 Bologna, Italy; Casari: Phone: +39-051-209-8662, Fax: +39-051-209-8493, marco.casari@unibo.it; Dragone: Phone: +39-051-209-8880, Fax: +39-051-209-8143, davide.dragone@unibo.it. We thank for comments Alberto Bisin, Tim Cason, Luigi Franzoni, Giuseppe Pignataro and the participants at the M-BEES 2010 in Maastricht, the EWEBE 2010 in Bertinoro, the IMEBE 2011 in Barcelona, the BEE 2011 in Florence, the SAET 2011 in Faro, the Max Planck Institute of Economics in Jena, Purdue University and University of Bologna. We also thank the composer Paolo Ingrosso for providing the noise for the experiment. This paper is part of the SONIC project at the University of Bologna. Financial support from AICCON, from the Italian Government (PRIN 2007/MCKEYA_003), and from the Max Planck Institute of Economics in Jena (ESI) is gratefully acknowledged. The usual disclaimer applies. 
Intertemporal choices are ubiquitous in economics, and understanding their motivations is crucial to predict patterns of retirement savings, purchase of durable goods, addictions, and health choices, just to name a few. The empirical literature has shown that impatience and temptation are major motivations of intertemporal choices (e.g., Schelling, 1978, Warner and Pleeter, 2001, Benartzi and Thaler, 2004, Brown et al., 2009, Tanaka et al., 2010). There may be other important drives such as risk, uncertainty, and anticipatory feelings. These drives have some bearing on the topic and may be an essential part of many intertemporal problems (Loewenstein and Prelec, 1991, Sozou, 1998, Fernandez-Villaverde and Mukherji, 2002, Dasgupta and Maskin, 2005, Halevy, 2008, Manzini and Mariotti, 2010). This study aims at uncovering their impact. The empirical issue is how to disentangle the role of anticipatory feelings and uncertainty from the role played by impatience and temptation. To this end, we present a model of intertemporal choice that extends the canonical model by incorporating uncertainty and anticipatory feelings. We also designed and ran an experiment where impatient agents are predicted to behave in a similar way, irrespective of whether they are exponential or quasi-hyperbolic discounters. Hence observed deviations from these predictions ought to originate from motivations other than impatience or temptation.

Interestingly, the vast majority of the experimental studies on time preferences have a static setting: subjects choose only at one date. This study presents an experiment of intertemporal choices in a dynamic setting where subjects choose at multiple dates, which allows for a deeper understanding of time preferences and, in particular, of choice reversals over time. Under the customary assumption of exponential time preferences, an individual should choose an optimal plan of consumption and then, given the same information, carry out the plan (Strotz, 1955). To validate this prediction, one should follow the individual choices over time or, at least, elicit the current plan and then track its future implementation. However, many experiments adopt a different technique to study time consistency: they compare a series of intertemporal choices made in a given moment. We refer to this as the static experimental approach. Such experiments often report a lower discount factor for short-run horizons than for long-run horizons, and then conclude that time preferences are not exponential (e.g., Kirby and Herrnstein, 1995). To account for this evidence, many scholars argue in favor of a quasi-hyperbolic model of time preferences as a better descriptor of intertemporal choices and suggest that individuals are tempted by, and some of them fall for, instantaneous gratification (e.g., Laibson, 1997). Instead, we adopt a dynamic 
experimental approach, which elicits individual decisions at multiple points in time. The dynamic approach allows tracking the consistency between a plan and its implementation, and it provides useful information for studying self-control problems.

In the experiment subjects faced a task to be completed under a deadline. The task required a real effort as it involved listening to annoying noise, which had to be "consumed" in the lab..$^{1}$ In a sense, it is prototypical of any activity requiring a costly effort. The basic elements of the experimental design are as follows. Each subject attended three sessions in exchange for a fixed participation fee.

In Session 0: Subjects rank three choices: to listen to the noise immediately $(\tau=0)$, in two weeks $(\tau=1)$, or in four weeks $(\tau=2)$. In addition, subjects bid in an auction to acquire the option to revise their ranking in two weeks, i.e. they could buy flexibility. All choices were properly incentivized.

In Session 1: All subjects who did not listen to the noise in Session 0 rank two options: to listen to the noise immediately $(\tau=1)$ or in two weeks $(\tau=2)$. This ranking of Session 1 applies if a subject won the auction to revise her ranking; otherwise, the ranking of Session 0 applies.

In Session 2: All subjects who did not listen to noise in either Session 0 or Session 1 listen to noise.

We report that about two thirds of subjects preferred to anticipate noise listening to session 0. Moreover, choice reversal was common even if it cannot be explained by temptation alone. This empirical evidence is not a falsification of the exponential or the quasi-hyperbolic models, but rather it strongly suggests the presence of other major motivations for intertemporal choices beyond impatience and temptation. In particular, the data points toward an important role for uncertainty and anticipatory feelings.

This paper contributes both to the experimental and the theoretical literature on intertemporal choices. First of all, it provides a model of intertemporal choice that extends the canonical discounting model by allowing for uncertainty and anticipatory feelings. The model clarifies the interwinding roles of discounting, sophistication and uncertainty for the demand for precommitment and flexibility. More generally, it provides a unified framework for issues that are often presented separately in the literature on intertemporal choice. It

\footnotetext{
${ }^{1}$ Using noise as a stimulus is common among experimental psychologists (e.g., Millar and Navarick, 1984).
} 
also puts forward different, complementary notions of choice reversals over time that are key to understand the issue of time inconsistency. In the second part, the paper presents a novel dynamic experiment that employs a consumption good, rather than money. Consumption goods are an important domain of choice for economic decisions and can solve methodological issues for the empirical measurement of time preferences, in particular storability and tradability. The experimental results show the existence of choice reversals even when present-biased preferences alone would not predict them. They also provide convincing evidence about the relevance of other drives beyond discounting.

The paper is structured as follows. Section 1 reviews the literature. Section 2 describes the theoretical setup and Section 3 describes the experimental design. Section 4 puts forward theoretical predictions for models driven by discounting, uncertainty, and anticipatory feelings. Section 5 presents the experimental results. Discussions and concluding remarks are presented in Sections 6 and 7.

\section{Literature review}

There are few dynamic experiments about time preferences: some studies concern choices about attendance at the gym and about physical exercise (Dellavigna and Malmendier, 2006, Charness and Gneezy, 2009), others consider studying activities (Ariely and Wertenbroch, 2002), or money (Casari, 2009). These studies provide evidence on how subjects dynamically implement their plan of actions over time. Most of them focus their analysis and discussions on the role of present-biased preferences and on the ability of a model based on exponential discounting to explain the evidence. Special attention is given to the agents' sophistication in intertemporal choices and to the role of precommitment devices. More generally, most of the current debate in intertemporal choices is about present-biased preferences (Laibson, 1997, O’Donoghue and Rabin, 1999). Instead, this paper aims at uncovering which factors may drive intertemporal choices besides discounting and temptation.

A key finding in favor of present-biased preferences is the widespread evidence of choice reversals over time (e.g. Kirby and Herrnstein, 1995, Benhabib et al., 2006, Casari, 2009). Some of the explanations for choice reversal refer to the temptation of immediate rewards or lack of self-control (e.g., Laibson, 1997, Fudenberg and Levine, 2006), while others rely on other drivers. A theoretically well-known driver is uncertainty about the future, which 
may be articulated in many ways. Yaari (1965) studies the impact of uncertain lifetime; Sozou (1995) considers discounting as a function of the risk that a delayed reward may not be received; Azfar (1999) studies the role of uncertain discount rates; Fernández-Villaverde and Mukherji (2006) consider random shocks on consumption preferences; Dasgupta and Maskin (2005) study an uncertain environment where payoffs may be realized early; and Halevy (2008) considers the role of uncertain lifetime and default probability. Interestingly, experimental results of intertemporal choices are often interpreted under the assumption of a certainty scenario. This assumption facilitates the identification of a point estimate for discount factors, but it neglects that uncertainty is often an intrinsic feature of intertemporal decisions, even when the experimenter does not explicitly introduce it. The neglect of uncertainty may be one of the sources of the large variability in the estimates of discount factors reported in the literature (Frederick et al., 2002). A way of dealing with both uncertainty and present-biased preferences is presented in O'Donoghue and Rabin (1999), who consider the case where the cost to complete a task is stochastic and agents may have incentives to procrastinate. Our theoretical set-up builds upon O'Donoghue and Rabin (1999), presents alternative definitions for choice reversals over time, and allows for discounting, uncertainty, and anticipatory feelings.

There exist experiments of intertemporal choice involving money and experiments involving non-monetary rewards. Both domains are relevant for economic decisions and both may help in studying time preferences. The former domain is relevant for financial decisions, while the latter one is relevant for economic situations involving, for instance, exerting effort, completing chores, or consuming goods. Experiments with non-monetary rewards have used noise (Millar and Navarick, 1984), rice (Pender, 1996), drinking water (Brown et al., 2009), and chocolate (Reuben et al., 2010). The most common practice among experimental economists is to use money as a reward. It is possible that the experimental results over money differ from those over a consumption good if preferences are domain-specific and/or if there are measurement errors when using money. In both cases additional evidence on consumption goods can shed light on time preferences. For instance, if time preferences turned out to be domain specific, the results obtained over money rewards would not extend to very common activities in the workplace and in everyday life such as filing a tax return, tiding up the office desk, completing an assignment, going to the gym, watching TV, or playing with the children. One aspect that may distinguish monetary from non monetary outcomes 
is the arousal of anticipatory feelings. Loewenstein (1987) argued that there may be anxiety while waiting for a bad outcome, and savouring while waiting for a positive outcome, in which cases anticipatory feelings produce an opposite effect with respect to discounting. The idea of anticipatory feelings is appealing and intuitive, however the existing evidence over intertemporal choices is still weak (Casari and Dragone, 2011) and deserves further experimental validation.

Another possible reason for domain-specific experimental results are measurement errors (Coller and Williams, 1999, Cubitt and Read, 2007). One issue with money is storability, which does not allow controlling for the timing of consumption. Instead, with a reward to be consumed in the lab and that cannot be stored for later, one can be sure about the timing of consumption and obtain more reliable information on time preferences. There is also an issue of tradability of money outside the lab, which allows for intertemporal arbitrage due to the existence of an external credit market. Both storability and tradability of the reward medium may bias the measurement of intertemporal preferences in the laboratory $\left.\right|^{2}$

\section{Completing a task under a deadline: a model}

This Section presents a model of intertemporal choice that includes discounting, anticipatory feelings, and stochastic utility as its basic components. First, it describes the utility function and the model setup, which is dynamic because agents make choices at multiple dates. Then, it studies how individual choices depend on whether the plan of actions is rigid or flexible.

\subsection{The utility function}

Consider a discrete-time environment, where $t \in\{0,1,2, \ldots, T\}$ and $T$ can be either finite or infinite. At time $\tau$ the agent consumes $x \in \mathbb{R}^{n}$ and obtains an instantaneous utility $u\left(x_{\tau}\right)=z_{\tau} \cdot v(x)$, where $z_{\tau} \in[0,+\infty]$ is a random shock and $v(x)$ is a stationary function. Assume that the agent's intertemporal utility is separable across periods and that it can be represented by the following weighted sum of current and future instantaneous utility

$$
U(t)=\sum_{\tau=t}^{T} \Phi(\tau-t) u\left(x_{\tau}\right) .
$$

\footnotetext{
${ }^{2}$ Some consumption goods like rice or chocolate do not solve the storability and tradability issues.
} 
The weight function $\Phi(\tau-t)$ depends on the delay between the consumption date $\tau$ and the decision date $t$. We consider the following separable weight function, for $\tau \geq t$ :

$$
\Phi(\tau-t)=\frac{D(\tau-t)+\mathbf{1}_{A} A(\tau-t)}{D(0)+\mathbf{1}_{A} A(0)} .
$$

The term $D(\tau-t)$ represents a discounting function where $D(\tau-t) \geq 0$ and $D^{\prime}(\tau-t) \leq 0$; $D(0)$ is normalized to one. The term $A(\tau-t)$ represents an anticipatory feelings function, with $A(\tau-t) \geq 0, A^{\prime}(\tau-t) \geq 0$, and $A(0)=1 . \mathbf{1}_{A}$ is an indicator function: it is equal to one if the good generates anticipatory feelings and it is zero otherwise. As a consequence $\Phi(\tau-t) \geq 0$ and $\Phi(0)=1$; the sign of $\Phi^{\prime}(\tau-t)$ can be either positive or negative, depending on the relative importance of discounting and anticipatory feelings. For goods that do not generate anticipatory feelings, the weight function boils down to a standard discounting function, i.e. $\Phi(\tau-t)=D(\tau-t) / D(0)$; as it is non increasing in the delay between the time of consumption and the current date, $\Phi(\tau-t)^{\prime} \leq 0$, receiving the good soon is (weakly) preferred to receiving it later. For goods that generate anticipatory feelings, instead, anticipating consumption may be suboptimal.

\subsection{A simple dynamic setup}

To study intertemporal choices it is useful to track individual choices as time goes on. This requires a dynamic setup where agents make decisions at multiple dates. We consider a simple case with three equally distant dates (0,1 and 2) and agents who make decisions at dates 0 and 1. Agents face three decision problems (Figure 1):

Decision A: at date 0 , choice between consuming $x_{0}$ or $x_{1}$;

Decision B: at date 0 , choice between consuming $x_{1}$ or $x_{2}$;

Decision C: at date 1 , choice between consuming $x_{1}$ or $x_{2}$.

Suppose that, when making her choice at date $t$, the agent knows the current realization of the random shock $z_{t}$. In decision $\mathrm{A}$, the agent prefers to consume immediately $x_{0}$, rather than consuming $x_{1}$ at date 1 , if

$$
u\left(x_{0}\right) \geq \Phi(1) \mathbf{E}\left[u\left(x_{1}\right)\right]
$$

where $\mathbf{E}\left[u\left(x_{1}\right)\right]$ is the expected utility of consuming $x_{1}$. In decision $\mathrm{B}$, the current realization of the shock does not matter; the agent prefers sooner consumption $x_{1}$ if

$$
\Phi(1) \mathbf{E}\left[u\left(x_{1}\right)\right] \geq \Phi(2) \mathbf{E}\left[u\left(x_{2}\right)\right]
$$




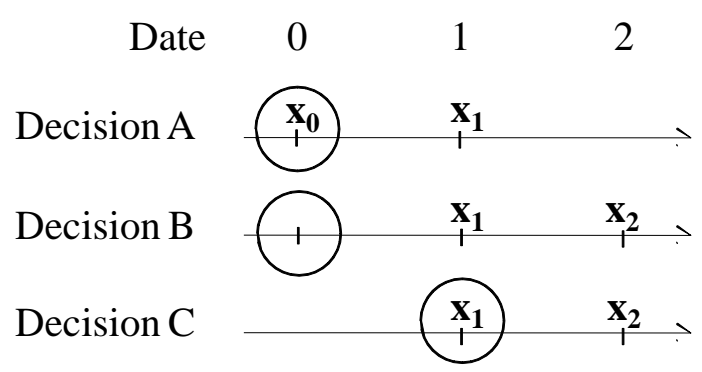

Figure 1: Decisions A, B, and C. The circle indicates when the choice is elicited. Comparison between $\mathrm{A}$ and $\mathrm{B}$ informs on static choice reversal, comparison between $\mathrm{B}$ and $\mathrm{C}$ on dynamic choice reversal, comparison between $\mathrm{A}$ and $\mathrm{C}$ on calendar choice reversal.

and she prefers later consumption $x_{2}$ otherwise. Decision $\mathrm{C}$ is similar to decision $\mathrm{A}$, with the only difference that all dates are shifted forward in time; the agent prefers to consume immediately $x_{1}$ if

$$
u\left(x_{1}\right) \geq \Phi(1) \mathbf{E}\left[u\left(x_{2}\right)\right]
$$

and she prefers to postpone and consume $x_{2}$ otherwise.

\subsection{Three notions of choice reversal}

A dynamic setup allows for studying different definitions of choice reversal. This is useful because the experimental literature defines choice reversal over time differently from the theoretical literature. Experimentalists generally infer choice reversal through comparisons across decision problems that are faced at one point in time. In the typical choice task, subjects are presented with two decision problems: one over a short-run horizon and another over a long-run horizon. For instance, they choose between receiving $\$ 100$ now and receiving $\$ 110$ tomorrow, and between receiving $\$ 100$ in 30 days and receiving $\$ 110$ in 31 days. If a subject chooses $\$ 100$ now in the former decision and $\$ 110$ in 31 days in the latter one, then a choice reversal is detected (Frederick et al., 2002). Theorists instead define choice reversal through comparisons within the same decision problem that is faced at different points in time (Strotz, 1955). Consider, for instance, a person deliberating whether to start dieting in March rather than April. If in January she prefers to start dieting in March and then in March she prefers to start in April, then theorists detect a choice reversal.

To characterize intertemporal choices and clarify the misunderstandings between the 
experimental and the theoretical literature, we propose three formal definitions: static, calendar, and dynamic choice reversal. These definitions are based on pairwise comparisons between the choices made in decisions A, B, and C (Figure 1), but they can be extended to a general intertemporal setup. Given our dynamic experimental design, we will be able to quantify choice reversals according to all three definitions.

Definition 1. A static choice reversal is detected if sooner consumption is chosen in A and later consumption in $\mathrm{B}$, or later consumption is chosen in $\mathrm{A}$ and sooner consumption in B.

Definition 2. A dynamic choice reversal is detected if either sooner consumption is chosen in B and later consumption in $\mathrm{C}$, or later consumption is chosen in $\mathrm{B}$ and sooner consumption in $\mathrm{C}$.

Definition 3. A calendar choice reversal is detected if either sooner consumption is chosen in $\mathrm{A}$ and later consumption in $\mathrm{C}$, or later consumption is chosen in $\mathrm{A}$ and sooner consumption in $\mathrm{C}$.

Only a dynamic experimental design that includes decision $\mathrm{C}$ enables us to measure all three notions of choice reversal. Experimentalists typically employ the notion of static choice reversal as evidence for a non constant discount rate. For studying self-control problems, however, one is interested in assessing whether the choice made under the temptation of immediate gratification (decision $\mathrm{C}$ ) is consistent with the plan made in advance (decision B), when the temptation was still distant in time. Hence, to study self-control and the role of flexibility and precommitment, dynamic choice reversal is a more useful notion than static choice reversal.

\subsection{Rigid and flexible plans for completing a task under a deadline}

Consider a task to be completed within a deadline. Completing the task at date $\tau$ entails a cost, $u\left(x_{\tau}\right)=-c_{\tau}$, where $c_{\tau}$ captures any immediate disutility to the agent arising from completing it, including opportunity cost. Let $c_{\tau}=z_{\tau} \cdot c$, where $z_{\tau}$ represents a stochastic shock that affects the cost to the agent of completing the task. The task cost is drawn from a stationary distribution with support $[\underline{\mathrm{c}}, \bar{c}]$, where $\underline{\mathrm{c}} \geq 0$, and cumulative distribution function $F\left(c_{\tau}\right)$. For notational simplicity, let $F(\underline{\mathrm{c}})=0$. We assume that the agent knows the density function $f\left(c_{\tau}\right)$ and that, at date $t$, she observes the task cost realization $c_{t}$ before choosing 
whether or not to perform the task. This uncertainty scenario is similar to O'Donoghue and Rabin (1999).

We consider the case where $T=2$. If the task is completed within date 2 , the agent is paid a fixed monetary amount $\bar{m}$ at date 2 and she is paid $\underline{m} \in[0, \bar{m})$ otherwise. $3^{3}$ Uncertainty does not affect the utility of money and future monetary rewards do not generate anticipatory feelings. By assuming separability between money and task cost, the agent's utility (1) from completing the task at time $\tau$ is,

$$
U(t)=\left\{\begin{array}{cc}
D(2-t) \bar{m}-c_{t} & \text { if } \tau=t \\
D(2-t) \bar{m}-\Phi(\tau-t) \mathbf{E}\left[c_{\tau}\right] & \text { if } \tau>t
\end{array}\right.
$$

where $\mathbf{E}\left[c_{\tau}\right]$ is the expected cost of completing at $\tau$. We assume that the expected cost is $c^{e}$ for all $\tau>t$, and that the agent always prefers completing the task rather than not completing it and receiving only $\underline{m}$.

The preference over completing the task at a specific date depends on whether the agent follows a rigid plan or a flexible plan.

Under rigid planning an agent makes a plan of actions at a specific date without the option of updating this plan in future dates. The agent's preferred date for completing the task is the one that yields the minimum expected cost. At date $t$, before choosing whether or not to perform the task, the agent observes the task cost realization $c_{t}$. Hence, at date $t$ the agent prefers completing the task immediately if the task cost is sufficiently low. We define $s_{\text {rigid }}^{t}$ as the cutoff value such that it is optimal for the agent to complete the task in the current date $t$ if and only if $c_{t} \leq s_{\text {rigid }}^{t}$, where for $t<T$ :

$$
s_{\text {rigid }}^{t}=\left\{\begin{array}{cc}
\psi_{t} & \text { if } \underline{\mathrm{c}} \leq \psi_{t} \leq \bar{c} \\
\underline{\mathrm{c}} & \text { if } \psi_{t}<\underline{\mathrm{c}} \\
\bar{c} & \text { if } \psi_{t}>\bar{c}
\end{array}\right.
$$

and

$$
\psi_{t}=\min _{\tau \in(t, T]}\{\Phi(\tau-t)\} c^{e} .
$$

The value $\psi_{t}$ is the minimum expected cost of completing the task at a future date.

\footnotetext{
${ }^{3}$ In O'Donoghue and Rabin (1999, p. 780), when the agent completes the task at date $t$, she receives a payment in the following date. Hence the terminal time is $T=t+1$ and is not fixed.
} 
In our setup, where $T=2$, when the agent makes a rigid plan at date 0 , the minimum expected cost to complete the task in a future date is: ${ }^{4}$

$$
\psi_{0}= \begin{cases}\Phi(1) c^{e} & \text { if } \Phi(1) \leq \Phi(2) \\ \Phi(2) c^{e} & \text { if } \Phi(1) \geq \Phi(2) .\end{cases}
$$

Now consider an agent who can update her initial plan of actions at any date $t$. We denote this case as flexible planning. The agent observes the task cost realization $c_{t}$ before choosing whether or not to perform the task. Optimality may require that the agent completes the task when the task cost is low, and that she waits when the task cost is high. Given a timehorizon $T$ within which the agent must complete the task, at time $t$ the agent's behavior can be described by a strategy $\mathbf{s}_{\text {flex }}^{t} \equiv\left(s_{t}^{t}, s_{t+1}^{t}, \ldots, s_{T}^{t}\right)$, which is a vector of cutoff costs such that the agent plans to complete the task at date $k=t, t+1, \ldots, T$ if and only if $c_{k} \leq s_{k}^{t} \in[\underline{\mathrm{c}}, \bar{c}] \mathrm{I}^{5}$ If the task has not been completed by date $T$, by assumption the agent completes it at $T$ irrespective of the realization of the $\operatorname{cost} c_{T}$, which implies $s_{T}^{t}=\bar{c}$ for all $t$. To compute the remaining cutoff values, we follow O'Donoghue and Rabin (1999) in defining a hazard function $h\left(\tau \mid t, \mathbf{s}_{\text {flex }}^{t}\right)$, which denotes the probability that the agent will not complete the task before date $\tau>t$ given that she has waited up to date $t$ and plans to follow strategy $\mathbf{s}_{\text {flex }}^{t}$ thereafter:

$$
h\left(\tau \mid t, \mathbf{s}_{\text {flex }}^{t}\right)=\left\{\begin{array}{cc}
1 & \text { if } \tau=t+1 \\
\prod_{i=t+1}^{\tau-1}\left(1-F\left(s_{i}^{t}\right)\right) & \text { if } \tau>t+1 .
\end{array}\right.
$$

From the perspective of date $t$, an agent who waits at date $k$ and then follows strategy $\mathbf{s}_{\text {flex }}^{t}$ thereafter incurs the following expected (weighted) task cost:

$$
\zeta_{k}\left(\mathbf{s}_{\mathrm{flex}}^{t}\right)=\sum_{\tau=k+1}^{T} \Phi(\tau-t) h\left(\tau \mid t, \mathbf{s}_{\mathrm{flex}}^{t}\right) F\left(s_{\tau}^{t}\right) \mathbf{E}\left[c_{\tau} \mid c_{\tau} \leq s_{\tau}^{t}\right],
$$

\footnotetext{
${ }^{4}$ If $\Phi(1)>\Phi(2)$, completing at date 2 is preferred to completing at date 1 because discounting weights more than anticipatory feelings (for events at dates 1 and 2 ). If instead $\Phi(1)<\Phi(2)$, the opposite holds. Which of the two conditions holds is revealed in decision B.

${ }^{5}$ If agents are aware of their future preferences (e.g. time consistent agents and sophisticated time inconsistent agents), beliefs over cutoff values used in future periods are correctly assessed, e.g. $s_{1}^{0}=s_{1}^{1}$. For time inconsistent agents, the cutoff costs may depend on when the flexible plan is elicited. For instance, at date 0 a naïve quasi-hyperbolic discounter has a belief $s_{1}^{0}$ about the cutoff value that will be used at date 1 , which may not be the actual cutoff value $s_{1}^{1}$ used at date 1 . Note that O'Donoghue and Rabin (1999) use a slightly different notation.
} 
where $\mathbf{E}[c \mid c \leq \alpha] \equiv \int_{\underline{c}}^{\alpha} c f(c) \mathrm{dc} / F(\alpha)$. From the perspective of date $t$, the agent should complete the task at date $k$ if the task $\operatorname{cost} c_{k}$ is less or equal to the expected cost of waiting at $k$, conditional on following strategy $\mathbf{s}_{\text {flex }}^{t}$ in the future. This implies that, for $t<T, s_{k}^{t}$ is:

$$
s_{k}^{t}=\left\{\begin{array}{cc}
\zeta_{k}\left(\mathbf{s}_{\text {flex }}^{t}\right) & \text { if } \underline{\mathrm{c}} \leq \zeta_{k}\left(\mathbf{s}_{\text {flex }}^{t}\right) \leq \bar{c} \\
\underline{\mathrm{c}} & \text { if } \zeta_{k}\left(\mathbf{s}_{\text {flex }}^{t}\right)<\underline{\mathrm{c}} \\
\bar{c} & \text { if } \zeta_{k}\left(\mathbf{s}_{\text {flex }}^{t}\right)>\bar{c} .
\end{array} .\right.
$$

In our setup, where $T=2$, the hazard function is $h\left(1 \mid 0, \mathbf{s}_{\text {flex }}^{0}\right)=h\left(2 \mid 1, \mathbf{s}_{\text {flex }}^{1}\right)=1$ and $h\left(2 \mid 0, \mathbf{s}_{\text {flex }}^{0}\right)=1-F\left(s_{1}^{0}\right)$. This implies the following:

$$
\begin{aligned}
& \zeta_{0}\left(\mathbf{s}_{\mathrm{flex}}^{0}\right)=\Phi(1) F\left(s_{1}^{0}\right) \mathbf{E}\left[c_{1} \mid c_{1} \leq s_{1}^{0}\right]+\Phi(2)\left(1-F\left(s_{1}^{0}\right)\right) c^{e}, \\
& \zeta_{1}\left(\mathbf{s}_{\mathrm{flex}}^{0}\right)=\Phi(2)\left(1-F\left(s_{1}^{0}\right)\right) c^{e}, \\
& \zeta_{1}\left(\mathbf{s}_{\mathrm{flex}}^{1}\right)=\Phi(1) c^{e} .
\end{aligned}
$$

The probability of completing the task increases as the deadline $T$ approaches for both exponential and quasi-hyperbolic agents with no anticipatory feelings: $\zeta_{0}\left(\mathbf{s}_{\text {flex }}^{0}\right)<\zeta_{1}\left(\mathbf{s}_{\text {flex }}^{1}\right)$. This property may not hold, however, for agents with anticipatory feelings (see the Appendix for details).

\subsection{The value of flexibility and precommitment}

At date 0 an agent values flexibility if she prefers a flexible plan to a rigid plan. The opposite happens when an agent values precommitment. The preference for flexibility or for precommitment is strictly linked to the notion of dynamic choice reversal, and it depends on several factors: the weighting function, the expected distribution of future costs of task completion, and the beliefs about the agent's future behavior.

Consider a rigid plan made at date 0 , and suppose that the agent does not complete the task immediately because the current cost is too high $\left(c_{0}>s_{\text {rigid }}^{0}\right)$. Hence she will complete the task either at date 1 or at date 2, depending on her plan (decision B). More precisely, she will complete at date 1 if $\Phi(1) \leq \Phi(2)$, and she will complete at date 2 otherwise. Hence the expected weighted cost $C_{\text {rigid }}(0)$ of completing the task under rigid planning is, from the 
perspective of date 0 :

$$
\begin{aligned}
C_{\text {rigid }}(0) & = \begin{cases}\Phi(2) c^{e} & \text { if } \Phi(1) \geq \Phi(2) \\
\Phi(1) c^{e} & \text { if } \Phi(1) \leq \Phi(2)\end{cases} \\
& =\psi_{0}
\end{aligned}
$$

Consider a flexible plan made at date 0 , and suppose that the agent does not complete the task immediately because the current cost is too high $\left(c_{0}>s_{0}^{0}\right)$. The agent believes that she will complete the task at date 1 depending on the cutoff value $s_{1}^{0}$. The probability that the task cost at date 1 is low is $F\left(s_{1}^{0}\right)$. Hence, the expected weighted cost $C_{\text {flex }}(0)$ of completing the task under flexible planning is, from the perspective of time 0 :

$$
\begin{aligned}
C_{\text {flex }}(0) & =\Phi(1) F\left(s_{1}^{0}\right) \mathbf{E}\left[c_{1} \mid c_{1}<s_{1}^{0}\right]+\Phi(2)\left(1-F\left(s_{1}^{0}\right)\right) c^{e} \\
& =\zeta_{0}\left(\mathbf{s}_{\text {flex }}^{0}\right) .
\end{aligned}
$$

Precommitment is preferred if $C_{\text {rigid }}(0)<C_{\text {flex }}(0)$, and flexibility is preferred otherwise. The difference $C_{\text {rigid }}(0)-C_{\text {flex }}(0)$ depends on three elements: the functional form of the weighting function $\Phi$, the agent belief about her future behavior $s_{1}^{0}$, and the expectation on future task costs. Proposition 1 considers the case where discounting plays a major role with respect to anticipatory feelings at dates 1 and 2 , i.e. $\Phi(1) \geq \Phi(2)$. This case includes also models without anticipatory feelings.

Proposition 1 Let $\Phi(1) \geq \Phi(2)$. At date 0, flexibility is valuable if and only if

$$
\Phi(2) c^{e}>\Phi(1) \mathbf{E}\left[c_{1} \mid c_{1}<s_{1}^{0}\right]
$$

precommitment is valuable if and only if

$$
\Phi(2) c^{e}<\Phi(1) \mathbf{E}\left[c_{1} \mid c_{1}<s_{1}^{0}\right]
$$

The proof is in the Appendix and here we provide the intuition. A rigid plan does not condition actions on the task cost realization, hence the expected task cost is constant, $c^{e}$. As $\Phi(1) c^{e} \geq \Phi(2) c^{e}$, at date 0 the agent prefers completing at date 2 rather than at date 1. Under flexible planning, instead, the expected cost of completing at date 1 is lower than at date 2 because it is conditional on being lower than the cutoff value $s_{1}^{0}$. If, from the perspective of date 0 , the conditional expected cost of completing the task at date 1 , 
$\Phi(1) \mathbf{E}\left[c_{1} \mid c_{1}<s_{1}^{0}\right]$, is lower than the unconditional expected cost at date $2, \Phi(2) c^{e}$, then flexibility is valuable. Otherwise, it is better to stick to the rigid plan and precommitment is valuable.

Interestingly, for exponential and quasi-hyperbolic discounters, flexibility is always valuable. This seems at odds with the idea that sophisticated agents with present-biased preferences may demand precommitment to avoid incurring in dynamic choice reversals, and that oftentimes flexibility may be undesirable (Schelling, 1978, among others). However, it is not the mere presence of present-biased preferences that may generate dynamic choice reversals, but also how the costs are distributed over time. In general, for some distributions of future costs an agent may prefer flexibility, while for others she may prefer precommitment. Instead, when the future expected costs are constant across dates, which is the case we are focusing on, the cutoff value $s_{1}^{0}$ is endogenously set in such a way that flexibility is always valuable (see the Appendix for the proof) $]^{6}$

Flexibility and precommitment may be valuable also for agents whose anticipatory feelings are relatively more important than discounting for events occurring at dates 1 and 2 , as stated below:

Proposition 2 Let $\Phi(1) \leq \Phi(2)$. At date 0, flexibility is valuable if and only if

$$
\Phi(2) c^{e}<\Phi(1) \mathbf{E}\left[c_{1} \mid c_{1}>s_{1}^{0}\right]
$$

precommitment is valuable if and only if

$$
\Phi(2) c^{e}>\Phi(1) \mathbf{E}\left[c_{1} \mid c_{1}>s_{1}^{0}\right]
$$

\section{Experimental design}

We recruited subjects for an experiment made of three sessions over a one-month period. These experimental sessions were run at two-week intervals at dates 0,1 , and 2 . The main decision was about when to listen to a 20-minute indivisible package of annoying noise?

\footnotetext{
${ }^{6}$ Moreover, as shown in the Appendix, a naïve quasi-hyperbolic discounter values flexibility more than a sophisticated quasi-hyperbolic discounter.

${ }^{7}$ Note that the sound was unpleasant, but it did not cause pain. The noise had to be listened to once, and the 20 minutes could not be spread over two or more sessions. Therefore, the concavity of the utility
} 
i.e., at date 0 , 1, or 2. Following participation in all three sessions, each subject received $€ 30$. Exactly 100 subjects started off the experiment: 40 under treatment 1 and 60 under treatment 2, which presents some differences in the procedure..$^{8}$ Unless otherwise noted, the description refers to treatment 2. We begin by describing the procedure for session 0 .

Session 0 included various color-coded parts: a ranking of the dates for noise listening (blue), an auction for flexibility (red), an auction for a dummy task (white), an auction for listening to additional noise at 0 (yellow), and a choice of exit penalty (green). In treatment 1, the actual order was white, yellow, blue, red, and green, while in treatment 2 it was white, blue, red, and green.

In the blue part, subjects expressed their preferences on when to listen to 20 minutes of noise. Before choosing, everyone listened to a 3-minute sample noise. The available dates were 0 (today), 1 (two weeks later) and 2 (four weeks later), which generated six possible rankings (Table 1). At the end of the session, a 10-face die was rolled for every participant to determine the date of noise listening, and depending on the die roll, subjects listened to noise. If session 0 was ranked as most preferred, then the probability of listening to noise at 0 (today) was 0.60 ; the probability was 0.10 when least preferred; and 0.30 otherwise. The timing of noise listening had no impact on the monetary payments. In treatment 1 , the random draw and noise listening took place right after the blue part. Note that there is a positive probability that the subject would listen to noise at any date. Each subject has the incentive to truthfully reveal the ranking of noise listening for all sessions, and not simply to reveal her most preferred date: she will benefit from selecting the highest probability for her most preferred date and the smallest probability for the least preferred one.

In the red part, participants were partitioned into groups of two and placed a bid to buy flexibility in a second-price auction. This flexibility option allows for updating the blue ranking two weeks later (date 1). The red coupons with the subjects' bids were publicly collected, randomly partitioned into pairs, and stapled. Subjects with the highest bid in their pair won the auction and paid the other bid amount in session 2. Auction results were function cannot have any impact on the optimal timing of consumption. Instead, intertemporal choices are usually elicited using larger rewards for later dates, which implies that, for a given discount factor, the optimal timing of consumption also depends on the concavity of the instantaneous utility function.

${ }^{8}$ Treatment 2 was longer and also included intertemporal decisions about monetary rewards. We have not yet analyzed choices over money. 


\begin{tabular}{cccccc}
\hline \hline Rank & $\begin{array}{c}\text { Most preferred date } \\
\text { for noise listening }\end{array}$ & $\begin{array}{c}\text { Intermediate } \\
\text { preference for noise } \\
\text { listening }\end{array}$ & $\begin{array}{c}\text { Least preferred date } \\
\text { for noise listening }\end{array}$ & $\begin{array}{c}\text { No. of } \\
\text { subjects } \\
\text { (frequency) }\end{array}$ & $\begin{array}{c}\text { Demand flexibility } \\
\text { for }\end{array}$ \\
\hline 210 & Four weeks later & Two weeks later & Today & $5(5.7 \%)$ & 2 \\
102 & Two weeks later & Today & Four weeks later & $8(9.2 \%)$ & 4 \\
012 & Today & Two weeks later & Four weeks later & $57(65.5 \%)$ & 16 \\
201 & Four weeks later & Today & Two weeks later & $2(2.3 \%)$ & 2 \\
120 & Two weeks later & Four weeks later & Today & $10(11.5 \%)$ & 2 \\
021 & Today & Four weeks later & Two weeks later & $5(5.7 \%)$ & 1 \\
\hline Total & & & & $87(100 \%)$ & $71(100 \%)$ \\
\hline \hline
\end{tabular}

Table 1: Ranking of preferred dates for noise listening, elicited in session 0.

Note: The table lists profiles as they appeared in the instructions of treatment 2. The order in the instructions of treatment 1 was $012,102,210,021,120$, and 201. We did not detect a bias in favor of the first profile listed in the table. Information on flexibility is available for $n=71$ subjects and is coded as positive if the bid was above 5 eurocents.

revealed in session 1 . This auction yields a measure of the willingness to pay for flexibility. Positive bids reveal a perceived uncertainty. Subjects from treatment 1 who had already listened to noise in session 0 skipped the red part.

To familiarize subjects with the auction format, an auction for a dummy task (white) preceded the red part. In the white part, participants stated their minimum compensation for going to a room located one floor below the lab and bring back a book. An experimenter publicly collected the bids, randomly partitioned and publicly stapled them in pairs. Subjects with the lowest bid in their pair won the auction and received the other bid amount at the end of the session. The results were shown on a screen, and the winners were publicly announced and immediately requested to fetch the book. The instructions explicitly mentioned the optimal strategy of revealing the true minimum compensation for the task. In treatment 1 , there were groups of four subjects.

Only treatment 1 had a yellow part with a second-price auction. Subjects bid for their willingness to listen to a 20-minute package of noise in a random group of four. The lowest bidder won the auction and listened to noise in session 0. Hence it was possible that in 
session 0 a subject listened twice to 20 minutes of noise, i.e., in the yellow and the blue parts. The rationale of the yellow part was to check whether noise was annoying, which it was $\sqrt[9]{5}$

If a subject dropped out during a session, she received a show-up fee (€3, 1 subject did drop out). In addition, a subject could refuse to listen to noise when asked to do so by the experimenters, i.e. to opt out. A subject that participated in all three sessions and opted out received $€ 30$ minus an exit penalty. In the green part, each subject chose her exit penalty from among $€ 15$, €20, €25, and $€ 30$. The instructions explained that raising the exit penalty could discourage opting out behavior and hence help in earning the full €30 participation fee. The exit penalty is a form of soft precommitment to listen to noise because it does not remove the option of refusing to listen when asked to do so. Instead, it lowers the temptation of not implementing a plan of action by making it less attractive not to listen to noise.

At the end, the winners of the white and yellow auctions were paid. In treatment 1 , a non-anonymous questionnaire had to be completed before payment.

Session 1 included a ranking of the dates for noise listening (blue) and a choice over opting out (green). All subjects listened to a 1-minute sample noise and then expressed their preferences on when to listen to 20-minutes of noise between date 1 (today) and 2 (two weeks later). The blue part was not completed by subjects who had listened to noise in session 0. Subjects learned the results of the auction for flexibility (red) after completing the blue part. For auction winners, the relevant preferences on the date of noise listening were those revealed in session 1; for everyone else the relevant preferences were those revealed in session 0. A subject listened to noise in session 1 with a probability 0.66 if session 1 was ranked as most preferred, 0.33 if least preferred ${ }^{10}$ In the green part, subjects had the option to refuse

\footnotetext{
${ }^{9}$ With convex disutility a subject prefers spreading two packages of noise in two distinct sessions rather than listening to both in the same session. Pilot experiments showed that the disutility of noise was indeed convex. Auction winners still had to listen to a 3-minute sample noise in the blue part. Non-winners could do any reading activity at their desk.

${ }^{10}$ In session 1 , any probability larger than 0.50 of listening in the most preferred date can truthfully elicit a subject's preference. Similarly, in session 0, as long as the highest probability of implementation corresponds to the most preferred date and the lowest probability corresponds to the least preferred date, the subject has the correct incentives to truthfully reveal her preferences. Hence, it is inconsequential for the stated rankings whether the probabilities of implementation differ across sessions.
} 
to listen to noise if asked to do so. Finally, a 6-face die was rolled for every participant to determine the date of noise listening, and depending on the die roll, subjects listened to noise.

Session 2 included a choice over opting out (green) and a questionnaire. Subjects who were not asked to listen to 20 minutes of noise in session 0 or 1 completed the green part and, when appropriate, listened to noise. All subjects then completed a questionnaire and received the participation fee net of the price paid by the winners of the auction for flexibility (red) and of the exit penalty paid by those who refused to listen to noise.

Subjects were recruited from the undergraduate population of the University of (omissis). All sessions were run on Wednesdays. In the invitation message, we asked subjects to bring reading material of their choice. In order to minimize attrition, the invitation message also specified that participation was required in all three sessions. This may have generated an

oversampling of more patient subjects, but our aim is not a quantitative measure of the discount factor.

Subjects were seated at computer terminals separated by partitions. No communication among subjects was allowed. Instructions were distributed and read aloud. All decisions were taken with pen and paper. The decisions concerning the different color-coded parts were written on coupons of the same color. To listen to noise, subjects wore high-fidelity headphones connected to the computer terminals. Including instruction reading, average session durations were 120, 120, and 75 minutes, respectively. For treatment 1 average session durations were about 75, 40, and 40 minutes, respectively. Overall, the average payment per subject was $30 €$.

\section{Predictions}

The theoretical model presented in Section 2 supplies a useful framework to discuss intertemporal choices, choice reversal and the demand for flexibility and precommitment. In order to provide benchmarks to evaluate the experimental results, we present predictions for three special cases where intertemporal choices depend only on one component of the general model. This allows understanding the impact of the three components of the general model: discounting, uncertainty, and anticipatory feelings. 
Prediction 1 (Discounting utility model). Consider an agent who discounts the future and has no anticipatory feelings, $\Phi(t-\tau)=D(t-\tau)$ for all $\tau \geq t$; let the task cost be certain and constant across sessions, $c_{\tau}=c$ for all $\tau$. Then the agent

a) chooses 210 in session 0, chooses 21 in session 1;

b) never exhibits dynamic, static and calendar choice reversal;

c) does not demand flexibility.

Prediction 2 (Anticipatory feelings model). Consider an agent that does not discount the future and has anticipatory feelings, $\Phi(t-\tau)=A(t-\tau)$ for all $\tau \geq t$; let the task cost be certain and constant across sessions, $c_{\tau}=c$ for all $\tau$. Then the agent

a) chooses 012 in session 0, chooses 12 in session 1;

b) never exhibits dynamic, static and calendar choice reversal;

c) does not demand flexibility.

Prediction 3 (Stochastic utility model). Consider an agent who does not discount the future, nor has anticipatory feelings, $\Phi(t-\tau)=1$ for all $\tau \geq t$. Let the task cost be uncertain and i.i.d. across sessions. Then the agent

a) chooses profile 012 or 021 in session 0 if the current cost is low, $c_{0}<c^{e}$, and chooses profile 210 or 120 if the current cost is high, $c_{0}>c^{e}$; chooses profile 12 in session 1 if the current cost is low, $c_{1}<c^{e}$, and chooses profile 21 if the current cost is high, $c_{1}>c^{e}$;

b) may exhibit static, dynamic, and calendar choice reversal;

c) demands flexibility.

\section{Results}

This section presents four results, namely on profiles for noise listening (Results 1 and 2), on choice reversals (Result 3), and on the demand for flexibility (Result 4). Exactly 100 participants initiated the experiment. Out of these, 12 subjects dropped out: 1 during session 0,8 between session 0 and session 1, and 3 between session 1 and session 2. Moreover, 1 subject paid the exit penalty and attended all three sessions. Our full sample is therefore made up of 87 fulfillers: subjects who attended all three sessions and listened to noise when asked to do so. Of this set of fulfillers, 40 listened to noise at date 0 . The restricted sample is made up of the remaining 47 subjects who did not listen to noise at date 0 and therefore 
revealed their preferences both at dates 0 and 1 under proper incentives. Unless otherwise noted, the results refer to the full sample of fulfillers $(\mathrm{N}=87)$.

Result 1 In session 0, about 71\% of subjects preferred to listen immediately; only 8\% of subjects preferred to listen in 4 weeks;

Table 1 provides support for Result 1. The evidence from choices in session 0 does not support Prediction 1. According to Prediction 1, the best choice for agents discounting the future is profile 210, which was chosen by only $5.7 \%$ of subjects. Hence exponential and quasi-hyperbolic discounting models explain only a small fraction of the preferences of subjects on when to listen to noise. On the contrary, $88.5 \%$ of the rankings revealed in session 0 are compatible with a stochastic utility model (77/87 obs., Prediction 3). A similar percentage is reported for the restricted sample (39/47 obs., 89\%). A model of anticipatory feelings predicts the choice of profile 012 , which was the most common one $(65.5 \%$ of choices, Prediction 2]. In sum, the observed choices over profiles suggest that both uncertainty and anticipatory feelings are possible motivations behind intertemporal choices (Predictions 2 and 3). In line with Result 1, we also report that in session 1 about $70 \%$ of the subjects preferred to listen immediately.

Result 2 In session 0, about 86\% of subjects preferred listening to noise in 2 weeks rather than in 4 weeks.

Support for this result is in Table 1. Result 2 is also at odds with Prediction 1 (discounting utility model) and it suggests that for intertemporal choices over noise, anticipatory feelings may play a role (Prediction 2). The next result concerns choice reversal. ${ }^{11}$

Result 3 About $53 \%$ of subjects exhibited some form of choice reversal.

Table 2 provides support for Result 3. When considering the restricted sample, about $40 \%$ of subjects exhibited static choice reversal, $40 \%$ exhibited calendar choice reversal, and $25 \%$ exhibited dynamic choice reversal. In our setup, present-biased preferences do not

\footnotetext{
${ }^{11}$ Static choice reversal occurs if one chooses either 021, 021, 120, or 102. Dynamic choice reversal occurs if one chooses $012,102,120$ in session 0 and then 21 in session 1, or 210, 201, 021 in session 0 and then 12 in session 1. Calendar choice reversal occurs if either 012, 021 or 201 is chosen in session 1 and then 21 in session 2, or 210, 201, or 210 in session 1 and then 12 in session 2 .
} 


\begin{tabular}{cccccc}
\hline \hline \multirow{2}{*}{ Rank } & \multicolumn{3}{c}{ Choice reversal } & No choice reversal & Total number \\
& Static & Calendar & Dynamic & & of subjects \\
\hline 210 & 0 & 2 & 2 & 3 & $5(10.6 \%)$ \\
012 & 0 & 4 & 4 & 19 & $23(48.9 \%)$ \\
Others & 19 & 13 & 6 & 0 & $19(40.4 \%)$ \\
\hline Total & $19(40.4 \%)$ & $19(40.4 \%)$ & $12(25.5 \%)$ & $22(46.8 \%)$ & $47(100 \%)$ \\
\hline \hline
\end{tabular}

Table 2: Static, dynamic, and calendar choice reversal.

Note: The restricted sample includes fulfillers who did not listen to noise in session $0(\mathrm{~N}=47)$.

In the larger sample of fulfillers $(\mathrm{N}=87), 25$ subjects exhibited static choice reversal.

generate choice reversal (Prediction 1). The widespread presence of choice reversal points toward a fundamental role of other factors beyond present-biased preferences. Anticipatory feelings is not one such factor: according to Prediction 2, subjects should never reverse their choices. Hence the majority of the choices (53\%) contradicts both Predictions 1 and 2.

By contrast, a stochastic utility model is compatible with the observed preference profiles and patterns of choice reversals (Prediction 3). Static and calendar choice reversals are found empirically to occur with similar frequencies. A Pearson Chi-square test indicates that there is a statistically significant relationship between those exhibiting static and calendar choice reversal: $13 / 19$ of the subjects exhibiting static choice reversal also exhibit calendar choice reversal, while $6 / 28$ subjects exhibiting no static choice reversal also exhibit calendar choice reversal $\left(68.4 \%\right.$ vs. $31.6 \%$, Pearson $\left.\chi^{2}(1)=10.38, p=0.001\right)$. In particular, evidence of calendar choice reversal strongly suggests a role for exogenous shocks on the subjective valuations of completing the task today versus completing it in two weeks.

Below we present the results on the demand for flexibility, which are based on the choices of 71 subjects.

Result 4 About $38 \%$ of subjects were willing to pay for flexibility.

In a certainty scenario, neither exponential, nor quasi-hyperbolic discounters, nor subjects with anticipatory feelings should demand flexibility (Predictions 1 and 2). On the contrary, flexibility has value in an uncertainty scenario where the task cost can vary. 
Subjects who plan to listen in session 0 should be less willing to pay for flexibility because they are less likely to listen to noise in future sessions. We find 17 subjects out of 48 with ranking 012 and 021 who demanded flexibility, versus 10 out of 23 with other rankings (35.4\% vs. $43.5 \%$ ). However, there is no statistically significant relationship between the demand for flexibility and the preference for listening in session 0 (Pearson $\chi^{2}(1)=0.43 p=0.51$, $n=71$ ). Moreover, we report a higher demand for flexibility among subjects who exhibited some form of choice reversal. There are 11 subjects who demanded flexibility out of the 25 who reversed their choices, and 7 subjects who demanded flexibility out of the 22 who never reversed their choices (44.0\% vs. 31.8\%, Pearson $\chi^{2}(1)=0.7349, p=0.39, n=47$ ). Choice reversals may originate from a variety of reasons, for instance the shape of the weight function or exogenous shocks. While the demand for flexibility is an ex-ante measure of uncertainty, calendar choice reversal best captures the ex-post impact of exogenous shocks at decision time. In the data, there is a weakly significant positive correlation among the two measures: there are 10 subjects who demanded flexibility out of the 19 who exhibited calendar choice reversal, and 8 out of 28 who did not reverse their choices $(52.6 \%$ vs. $28.6 \%$, Pearson $\left.\chi^{2}(1)=2.77, p=0.10\right)$. A model of stochastic utility is compatible with these patterns of behavior 12

The impact of individual characteristics is reported in Casari and Dragone (2010). Gender did not play a significant role in any of the above choices while in some of them cognitive abilities, major, and smoking habits played a role. Subjects with higher cognitive abilities were more likely to demand flexibility. The results do not seem an artefact of the lack of economic training, as subjects with humanities majors were more likely to behave according to the discounting model (Prediction 1).

\section{Discussion}

Four aspects of the reported evidence deserve some comments. First, a large majority of subjects chose to anticipate noise listening and to avoid doing it at the last available date, which runs contrary to predictions of models based only on discounting. The magnitude

\footnotetext{
${ }^{12}$ Only $22.5 \%$ (16/71) increased the exit penalty. This evidence does not contradict Predictions 1 and 2, although no quantitative prediction could be made. Half of the 71 subjects demanded neither flexibility nor an increase in the exit penalty.
} 
of this finding was unexpected $(48 / 71=67.6 \%$ of subjects $)$ and may be explained through anticipatory feelings and stochastic utility. In particular, if subjects experience dread or anxiety while waiting for the moment of noise listening, anticipating it may be optimal (Prediction 2). When confronted with the data, a model of anticipatory feelings explains about one third of the individual choices elicited at date $0(28 / 71=39.4 \%$ of subjects $)$. The choice of anticipating noise listening is not related with experimental measures of cognitive abilities. To the best of our knowledge, this is the first incentivized experiment that shows a role for anticipatory feelings in intertemporal choices over non monetary outcomes $[13$ Below we will discuss the role played by uncertainty about the future task costs.

Second, about half of the subjects reversed their choices according to at least one of our definitions. In our setup, present-biased preferences alone cannot lead to choice reversals. More generally, within a certainty scenario choice reversal is not predicted by single-drive models based on discounting or on anticipatory feelings. Instead, a model of stochastic utility can go a long way in explaining the data because it encompasses anticipation of noise listening, choice reversal, and demand for flexibility. The key role of uncertainty in intertemporal choices is revealed by two cues. One is demand for flexibility, which is direct evidence that the subject fears future shocks. The other is dynamic choice reversal, which may occur in case of unpredicted events. In the restricted sample, 27 out of 47 subjects either demanded flexibility or displayed dynamic choice reversal (57\%), which points toward a substantive impact of uncertainty in intertemporal choices.

Third, in this study the canonical models of exponential or quasi-hyperbolic discounting lack descriptive power (Prediction 1). In these models, the best option for noise listening is the latest available date and the worst option is the earliest available date. Moreover, they predict no demand for flexibility and no choice reversal. Only 3 out of 71 subjects fit these predictions, which accounts for less than $5 \%$ of the observations. Hence, the empirical evidence does not support an explanation that relies on discounting as the only drive. This is not a statement that undermines the validity of models of discounting; rather, as we have seen, it suggests that uncertainty and anticipatory feelings also contribute to explain intertemporal choices.

Fourth, the experiment involved choices over a real effort task, i.e. listening to annoying

\footnotetext{
${ }^{13}$ Loewenstein (1987) presents suggestive evidence of anticipatory feelings in various domains. The evidence is based on hypothetical choices, which may not be robust to replication (Casari and Dragone, 2011).
} 
noise. Non-monetary choices are very relevant for a wide array of field decisions, and there exists a rich theoretical literature about non-monetary choices (e.g. O'Donogue and Rabin, 1999). Yet, experiments with this feature are rare in the economic literature (for an example concerning real effort, see Falk and Ichino, 2006). We provide novel, incentivized evidence on a real effort task that is complementary to the existing studies based on monetary rewards. Moreover, our design has the methodological advantage of addressing serious concerns related to the storability and tradability of monetary rewards (Cubitt and Read, 2007) and to exhibit predictions that do not depend on the concavity of the instantaneous utility function, nor on the shape of the discounting function.

There is also an issue related to the costly effort in completing the task, which confronts subjects with choices over losses. One may wonder if our results would extend to decisions over gains. We acknowledge that the size of the effects we report may be task-specific. It can be the case, for instance, that noise induces stronger anticipatory feelings than, say, a massage, which would imply that our evidence over-estimates the importance of anticipatory feelings. Unlike other studies, however, our aim is not a calibration of the discount factor, nor the exact assessment of the quantitative impact of the various drives behind intertemporal choices. Our goal is to provide reliable evidence about the existence of stochastic utility and anticipatory feelings as driving forces behind intertemporal choices. Hence, for our conclusions the crucial design feature is the real-effort nature of the task, which is particularly relevant if monetary rewards somehow "sterilize" the role of stochastic utility and anticipatory feelings. We leave it to further experimental studies the investigation of other tasks and rewards and to assess whether a similar magnitude of the reported findings may generalize to other scenarios.

\section{Conclusions}

We have studied time preferences and time consistency in an experiment that elicited a subject's plan and then tracked its implementation over time. More specifically, this paper makes three main contributions. First, it documents in a controlled, incentivized setting a widespread preference for completing a costly task immediately. Second, it offers explanations for these experimental results at the individual level. Third, it provides a systematic analysis of time inconsistency through novel definitions and measurements of choice reversals. 
The experiment presents a highly innovative design where subjects faced a decision between completing a given task sooner or later. It is the simplest design we could think of to study intertemporal choices. In most intertemporal choice experiments, the design usually requires choices over two dimensions: amount and timing of the reward. Typically, subjects face a decision between a smaller-sooner reward and a larger-later reward. In our setting, there is no trade-off between amount and timing of consumption because the only dimension which is relevant for the subjects is timing. As a consequence, the predictions on the optimal choice are straightforward: any participant who discounts the future wishes to bring forward rewards to an earlier point in time and defer costs to a later point in time.

In the experiment, choices were made over a one-month horizon and had a dynamic structure in the sense that subjects made decisions at multiple points in time, i.e., today and then again two weeks later. We report that two thirds of the subjects prefer to complete a costly task immediately, instead of postponing it at the last available date. This overwhelming evidence of a preference for anticipating a costly task comes from a properly incentivized experiment. Exponential or quasi-hyperbolic discounting are at odds with such evidence. The empirical evidence is compatible with economic theory if one is willing to admit stochastic utility and anticipatory feelings as factors affecting intertemporal preferences. We consider a set of simple models and for each one we put forward predictions on the optimal timing of consumption, demand for flexibility, and possible choice reversal. More specifically, between $53 \%$ and $69 \%$ of subjects fit the predictions of either a stochastic utility or an anticipatory feelings model. Less than $5 \%$ of subjects fit the predictions of exponential or quasi-hyperbolic discounting models. However, this evidence does not suggest that impatience and temptation play no role in intertemporal choices. Their role has already been convincingly documented in several empirical studies. As the design is such that exponential and quasi-hyperbolic discounters are predicted to behave in similar ways, any deviation from these predictions simply suggests that discounting is not the only drive affecting intertemporal choices. Our conclusion is that the canonical exponential discounting model or the quasi-hyperbolic discounting cannot fit the empirical evidence, and additional drives must be taken into account to understand intertemporal behavior.

Another contribution of this study concerns time inconsistency. Static choice reversal occurred for about a third of the individuals. This result is novel because it occurred without being triggered by temptation, showing that - besides present-biased preferences - there also 
exist other potential causes for choice reversals. In addition, we measure choice reversal in a dynamic way. The literature on time consistency, as well as most anecdotes about selfcontrol, relies on subjects taking decisions at multiple points in time. By contrast, the majority of experimental studies rely on a one-point measurement of time preferences. This static measurement captures some relevant aspects of the issue, although it differs from the dynamic measurement studied by, e.g., Strotz (1955). Importantly, the presence of static choice reversal does not necessarily imply the presence of dynamic choice reversal, nor vice versa. We can measure the frequency of dynamic choice reversal in the data because of our multi-stage design. We report that several subjects exhibit dynamic choice reversal over time. Its frequency is comparable to the frequency of static choice reversal, although the two types of choice reversal are not necessarily driven by the same factors.

Empirical studies of time preferences are methodologically difficult because of the intermingling of impatience, temptation, risk, uncertainty, and anticipatory feelings. This study aims neither at obtaining quantitative estimates of discount factors nor at disentangling exponential from quasi-hyperbolic discounting. Instead, the aim is to discover if there are other motivations for intertemporal choices besides impatience and temptation. With its dynamic design and real-effort task, this study takes a step toward understanding intertemporal choices. The evidence calls for models of intertemporal choice with a wider range of motivations, where impatience and temptation are complemented by stochastic utility and anticipatory feelings.

\section{References}

[1] Ariely, D., and Wertenbroch, K. (2002). "Procrastination, Deadlines, and Performance: Self-control by Precommitment". Psychological Science, 13, 219-224.

[2] Azfar, O. (1999). "Rationalizing Hyperbolic Discounting." Journal of Economic Behavior and Organization, 38, 245-252.

[3] Benartzi, S., and Thaler, R. H. (2004). "Save More Tomorrow: Using Behavioral Economics to Increase Employee Saving." Journal of Political Economy, 112, S164-S187. 
[4] Benhabib, J., Bisin, A., and Schotter, A. (2006). Present-Bias, Quasi-Hyperbolic Discounting, and Fixed Costs, working paper, New York University

[5] Brown, A. L., Chua Z. E., and Camerer, C. F. (2009). "Learning and Visceral Temptation in Dynamic Saving Experiments." The Quarterly Journal of Economics, 124, 197-223.

[6] Casari, M. (2009). "Precommitment and Flexibility in a Time-decision Experiment." Journal of Risk and Uncertainty, 38, 117-141.

[7] Casari, M., and Dragone, D. (2010). "Impatience, Anticipatory Feelings and Uncertainty: A Dynamic Experiment on Time Preferences." Jena Economic Research Papers 2010-087, Schiller-University of Jena, Max Planck Institute of Economics.

[8] Casari, M., and Dragone, D. (2011). "On Negative Time Preferences." Economics Letters, $111,37-39$.

[9] Charness, G., and Gneezy, U. (2009). "Incentives to Exercise." Econometrica, 77, 909931.

[10] Coller, M., and Williams, M. B. (1999). "Eliciting Individual Discount Rates". Experimental Economics, 2, 107-127.

[11] Cubitt, R. P., and Read, D. (2007). "Can Intertemporal Choice Experiments Elicit Time Preferences for Consumption?" Experimental Economics 10, 369-389

[12] Dasgupta, P., and Maskin, E. (2005). "Uncertainty and Hyperbolic Discounting." American Economic Review, 95, 1290-1299.

[13] Dellavigna, S., and Malmendier, U. (2006) "Paying Not To go To The Gym." American Economic Review, 96, 694-719.

[14] Falk, A., and Ichino, A. (2006). "Clean Evidence on Peer Effects". Journal of Labor Economics, 24, 39-58.

[15] Frederick, S., Loewenstein, G., and O'Donoghue, T. (2002). "Time Discounting and Time Preference: A Critical Review." Journal of Economic Literature, 15, 351-401. 
[16] Fundenberg, D., and Levine, D. K. (2006). "A Dual Model of Impulse Control." American Economic Review, 96, 1449-1476.

[17] Halevy, Y. (2008). "Strotz meets Allais: Diminishing impatience and the certainty effect." American Economic Review, 98, 1145-1162.

[18] Kirby, K. N., and Herrnstein, R.J. (1995). "Preference Reversals due to Myopic Discounting of Delayed Reward." Psychological Science, 6, 83-89.

[19] Laibson, D. (1997). "Golden Eggs and Hyperbolic Discounting." Quarterly Journal of Economics, 112, 443-77.

[20] Loewenstein, G. (1987). "Anticipation and the Valuation of Delayed Consumption." Economic Journal, 97, 666-84.

[21] Loewenstein, G., and Prelec, D. (1991). "Negative Time Preference." American Economic Review, 81, 347-352.

[22] Manzini, P., and Mariotti, M. (2010). "Moody Choice." IZA Discussion Paper 5005.

[23] Millar, A., and Navarick, D.J. (1984). "Self-control and Choice in Humans: Effects of Video Game Playing as a Positive Reinforcer." Learning and Motivation, 15, 203-218.

[24] O'Donoghue, T., and Rabin, M. (1999). "Incentives for Procrastinators." Quarterly Journal of Economics, 114, 769-816.

[25] Pender, J. L. (1996). "Discount Rates and Credit Markets: Theory and Evidence from Rural India." Journal of Development Economics, 50, 257-296.

[26] Reuben, E., Sapienza, P., and Zingales, L. (2010). "Time Discounting for Primary and Monetary Rewards." Economics Letters, 106, 125-127

[27] Schelling, T. C. (1978). "Egonomics, or the Art of Self-Management." American Economic Review, 68, 290-294.

[28] Sozou, P. D. (1998). "On Hyperbolic Discounting and Uncertain Hazard Rates." Proceedings of the Royal Society of London: Biological Sciences (Series B), 265 (1409), 2015-2020. 
[29] Strotz, R. H. (1955-1956). "Myopia and Inconsistency in Dynamic Utility Maximization." Review of Economic Studies, 23, 165-80.

[30] Tanaka, T., Camerer, C., and Nguyen, Q. (2010). "Risk and Time Preferences: Linking Experimental and Household Survey Data from Vietnam." American Economic Review, $100,557-571$.

[31] Warner, J. T. and Pleeter, S. (2001). "The Personal Discount Rate: Evidence from Military Downsizing Programs." American Economic Review, 91, 33-53.

[32] Yaari, M. (1965). "Uncertain Lifetime, Life Insurance, and the Theory of the Consumer." Review of Economic Studies, 32, 137-150. 


\section{Appendix}

A1. Under flexible planning, for exponential and quasi-hyperbolic agents the expected cost of waiting increases as the deadline approaches: $\zeta_{1}\left(\mathbf{s}_{\mathrm{flex}}^{1}\right)>\zeta_{0}\left(\mathbf{s}_{\mathrm{flex}}^{0}\right)$. This property does not hold for a generic weight function $\Phi$.

The expected weighted task costs are:

$$
\begin{aligned}
& \zeta_{0}\left(\mathbf{s}_{\text {flex }}^{0}\right)=\Phi(1) F\left(s_{1}^{0}\right) \mathbf{E}\left[c_{1} \mid c_{1}<s_{1}^{0}\right]+\Phi(2)\left(1-F\left(s_{1}^{0}\right)\right) c^{e} ; \\
& \zeta_{1}\left(\mathbf{s}_{\text {flex }}^{1}\right)=\Phi(1) c^{e} .
\end{aligned}
$$

If $F\left(s_{1}^{0}\right)=1$, then

$$
\zeta_{1}\left(\mathbf{s}_{\text {flex }}^{1}\right)-\zeta_{0}\left(\mathbf{s}_{\text {flex }}^{0}\right)=\Phi(1)\left(c^{e}-\mathbf{E}\left[c_{1} \mid c_{1}<s_{1}^{0}\right]\right) \geq 0 .
$$

If $F\left(s_{1}^{0}\right)<1$, then

$$
\begin{aligned}
\zeta_{1}\left(\mathbf{s}_{\mathrm{flex}}^{1}\right)-\zeta_{0}\left(\mathbf{s}_{\mathrm{flex}}^{0}\right) & =\Phi(1) c^{e}-\Phi(1) F\left(s_{1}^{0}\right) \mathbf{E}\left[c_{1} \mid c_{1}<s_{1}^{0}\right]-\Phi(2)\left(1-F\left(s_{1}^{0}\right)\right) c^{e} \\
& =\Phi(1)\left[\int_{\underline{c}}^{\bar{c}} x f(x) d x-\int_{\underline{\underline{c}}}^{s_{1}^{0}} x f(x) d x\right]-\Phi(2)\left(1-F\left(s_{1}^{0}\right)\right) c^{e} \\
& =\Phi(1) \int_{s_{1}^{0}}^{\bar{c}} x f(x) d x-\Phi(2)\left(1-F\left(s_{1}^{0}\right)\right) c^{e}
\end{aligned}
$$

which implies

$$
\frac{\zeta_{1}\left(\mathbf{s}_{\mathrm{fex}}^{1}\right)-\zeta_{0}\left(\mathbf{s}_{\mathrm{flex}}^{0}\right)}{1-F\left(s_{1}^{0}\right)}=\Phi(1) \mathbf{E}\left[c_{1} \mid c_{1}>s_{1}^{0}\right]-\Phi(2) c^{e} .
$$

Hence

$$
\zeta_{1}\left(\mathbf{s}_{\mathrm{flex}}^{1}\right)>\zeta_{0}\left(\mathbf{s}_{\mathrm{flex}}^{0}\right) \Leftrightarrow \Phi(1) \mathbf{E}\left[c_{1} \mid c_{1}>s_{1}^{0}\right]>\Phi(2) c^{e}
$$

Note that $\mathbf{E}\left[c_{1} \mid c_{1}>s_{1}^{0}\right] \in\left[c^{e}, \bar{c}\right]$ for all $s_{1}^{0}$. If there are no anticipatory feelings, then $\Phi(2) \leq$ $\Phi(1)$ and expression (13) always holds. In particular, it always holds for agents discounting the future exponentially or quasi-hyperbolically, irrespective of whether they are sophisticated or naïve quasi-hyperbolic discounters (i.e. irrespective of the belief on the cutoff value $\left.s_{1}^{0}\right)$.

While expression (13) may hold for agents with mild anticipatory feelings, it may not hold for agents with strong anticipatory feelings. Consider for example the case where $\Phi(\cdot)=A(\cdot)$ and a sufficiently high anticipatory feeling such that $A(2)>\mathbf{E}\left[c_{1} \mid c_{1}>s_{1}^{0}\right] A(1) / c^{e}$. In such a case $\zeta_{1}\left(\mathbf{s}_{\text {flex }}^{1}\right)<\zeta_{0}\left(\mathbf{s}_{\text {flex }}^{0}\right)$, which implies that the cutoff values of a flexible plan decrease as the deadline approaches. 


\section{A2. Optimal rankings in the general model}

The optimal strategy of the agent depends on whether the plan is rigid or flexible. Under a rigid plan established in session 0 , listening to noise in session 0 is preferred if and only if the current cost is lower than the minimum expected cost in sessions 1 and 2 ,

$$
c_{0} \leq \psi_{0}=\min \{\Phi(1), \Phi(2)\} c^{e} .
$$

Moreover, the optimal plan for the future sessions prescribes listening to noise in session 1 over session 2 if

$$
\Phi(1)<\Phi(2) .
$$

Hence the optimal ranking assessed in session 0 under rigid planning is:

$$
\begin{array}{ll}
210 & \text { if } \Phi(2) \leq \Phi(1) \leq c_{0} / c^{e} ; \\
201 & \text { if } \Phi(2) \leq c_{0} / c^{e} \leq \Phi(1) ; \\
021 & \text { if } c_{0} / c^{e} \leq \Phi(2) \leq \Phi(1) ; \\
012 & \text { if } c_{0} / c^{e} \leq \Phi(1) \leq \Phi(2) ; \\
102 & \text { if } \Phi(1) \leq c_{0} / c^{e} \leq \Phi(2) ; \\
120 & \text { if } \Phi(1) \leq \Phi(2) \leq c_{0} / c^{e} .
\end{array}
$$

An agent who is perfectly patient and has no anticipatory feelings $(\Phi(1)=\Phi(2)=1)$ may choose any plan, with the caveat that plans 102 and 201 should be chosen only if $c_{0}=c^{e}$. If $\Phi(2)<\Phi(1)$ (because, e.g., $\Phi(\cdot)=D(\cdot)$ ), the participant should choose either plan 021, 201, or 210. If $\Phi(2)>\Phi(1)$ (because, e.g., $\Phi(\cdot)=A(\cdot)$ ), the participant should choose either plan 012,102 , or 120 .

\section{A3. Value of flexibility}

Flexibility is valuable if the weighted expected cost of a flexible plan is lower than that of a rigid plan. We now compute the value of flexibility for the general case where the plan is implemented probabilistically ${ }^{14}$

Consider a person who makes a bid for flexibility at date 0 after she knows she will not listen to the noise in session 0 (treatment 1 ). Let $p>1 / 2$ be the probability of listening to the noise in session 1 when session 1 is preferred to session 2 or, alternatively, of listening in

\footnotetext{
${ }^{14}$ If the expected cost of a rigid plan is larger than that of a flexible plan, analogous considerations hold for the demand for precommitment.
} 
session 2 when session 2 is preferred to session 1 (in our design, $p=2 / 3$ ). At session 0 , the expected weighted cost of listening in future sessions under rigid planning is

$$
C_{\text {rigid }}(0)= \begin{cases}{[(1-p) \Phi(1)+p \Phi(2)] c^{e}} & \text { if } \Phi(1)>\Phi(2) \\ {[p \Phi(1)+(1-p) \Phi(2)] c^{e}} & \text { if } \Phi(1)<\Phi(2)\end{cases}
$$

At session 0 , the expected weighted cost of listening in future sessions under a flexible scenario is

$$
\begin{aligned}
C_{\text {flex }}(0)= & \Phi(1)\left\{p F\left(s_{1}^{0}\right) \mathbf{E}\left[c_{1} \mid c_{1}<s_{1}^{0}\right]+(1-p)\left(1-F\left(s_{1}^{0}\right)\right) \mathbf{E}\left[c_{1} \mid c_{1}>s_{1}^{0}\right]\right\} \\
& +\Phi(2)\left[(1-p) F\left(s_{1}^{0}\right)+p\left(1-F\left(s_{1}^{0}\right)\right)\right] c^{e} \\
= & (2 p-1) F\left(s_{1}^{0}\right)\left\{\Phi(2) c^{e}-\Phi(1) \mathbf{E}\left[c_{1} \mid c_{1}<s_{1}^{0}\right]\right\} .
\end{aligned}
$$

The maximum willingness to pay for flexibility is given by $C_{\text {rigid }}-C_{\text {flex }}$. Depending on whether there is a major role of discounting over anticipatory feelings, we have two possible cases:

$$
C_{\text {rigid }}(0)-C_{\text {flex }}(0)=\left\{\begin{array}{cl}
(2 p-1) F\left(s_{1}^{0}\right)\left[\Phi(2) c^{e}-\Phi(1) \mathbf{E}\left[c_{1} \mid c_{1}<s_{1}^{0}\right]\right] & \text { if } \Phi(1)>\Phi(2) \\
(2 p-1)\left(1-F\left(s_{1}^{0}\right)\right)\left[\Phi(1) \mathbf{E}\left(c_{1} \mid c_{1}>s_{1}^{0}\right)-\Phi(2) c^{e}\right] & \text { if } \Phi(1)<\Phi(2)
\end{array}\right.
$$

As the payment for flexibility occurs in session 2 and the value of flexibility is elicited in session 0 , the willingness to pay $M\left(s_{1}^{0}\right)$ is

$$
M\left(s_{1}^{0}\right)=\frac{C_{\text {rigid }}-C_{\text {flex }}}{D(2)} .
$$

Hence, in treatment 1 , when $\Phi(2) \leq \Phi(1)$ the agent is willing to pay to switch from a rigid to a flexible plan at most

$$
\max \left\{0, \frac{(2 p-1) F\left(s_{1}^{0}\right)}{D(2)}\left(\Phi(2) c^{e}-\Phi(1) \mathbf{E}\left[c_{1} \mid c_{1}<s_{1}^{0}\right]\right)\right\},
$$

which implies that flexibility is valuable if

$$
\frac{\Phi(2)}{\Phi(1)} \in\left(\frac{\mathbf{E}\left[c_{1} \mid c_{1}<s_{1}^{0}\right]}{c^{e}}, 1\right] .
$$

If instead $\Phi(2) \geq \Phi(1)$, the agent is willing to pay to switch from a rigid to a flexible plan at most

$$
\max \left\{0, \frac{(2 p-1)\left(1-F\left(s_{1}\right)\right)}{D(2)}\left[\Phi(1) \mathbf{E}\left(c_{1} \mid c_{1}>s_{1}\right)-\Phi(2) c^{e}\right]\right\}
$$


which implies that flexibility is valuable if

$$
\frac{\Phi(2)}{\Phi(1)} \in\left[1, \frac{\mathbf{E}\left[c_{1} \mid c_{1}>s_{1}^{0}\right]}{c^{e}}\right) .
$$

Overall, in treatment 1 flexibility is valuable if

$$
\frac{\Phi(2)}{\Phi(1)} \in\left(\frac{\mathbf{E}\left[c_{1} \mid c_{1}<s_{1}^{0}\right]}{c^{e}}, \frac{\mathbf{E}\left[c_{1} \mid c_{1}>s_{1}^{0}\right]}{c^{e}}\right)
$$

Now consider treatment 2, where the subjects make their bids to buy the flexibility option before they know whether they will listen to the noise in session 0 . Let $q>0$ be the probability that they do not listen to the noise in session 0 (in our setup, it could be either $4 / 10,7 / 10$ or $9 / 10$, depending on whether listening in session 0 was ranked as the best, intermediate or worst option, respectively). In treatment 2 the value of flexibility is $q M\left(s_{1}^{0}\right)$. Hence it is the lowest, everything else equal, if the participant chooses 012 or 021.

A4. If $\mathbf{E}\left[c_{1}\right]=\mathbf{E}\left[c_{2}\right]=c^{e}$, for exponential and quasi-hyperbolic discounters the value of flexibility is always positive. If $c_{1}=\mathbf{E}\left[c_{1}\right] \neq \mathbf{E}\left[c_{2}\right]=c_{2}$, quasi-hyperbolic discounters may value flexibility or precommitment depending on the expectations on the task cost distribution.

Let $\mathbf{E}\left[c_{1}\right]=\mathbf{E}\left[c_{2}\right]=c^{e}$. For agents discounting the future, the relevant condition for assessing whether flexibility is valuable is (see expression 17):

$$
\Phi(2) c^{e}-\Phi(1) \mathbf{E}\left[c_{1}<s_{1}^{0}\right]=\Phi(2) c^{e}-\Phi(1) \frac{\int_{\underline{\underline{c}}}^{s_{1}^{0}} x f(x) d x}{\int_{\underline{\underline{c}}}^{s_{1}^{0}} f(x) d x}>0
$$

If $F\left(s_{1}^{0}\right)>0$, then

$$
\Phi(2) c^{e} \int_{\underline{\underline{c}}}^{s_{1}^{0}} f(x) d x-\Phi(1) \int_{\underline{\underline{c}}}^{s_{1}^{0}} x f(x) d x=\int_{\underline{\underline{c}}}^{s_{1}^{0}}\left[\Phi(2) c^{e}-\Phi(1) x\right] f(x) d x .
$$

A sufficient condition for 20 to be positive is

$$
\Phi(2) c^{e}-\Phi(1) s_{1}^{0} \geq 0
$$

which implies

$$
\frac{\Phi(2)}{\Phi(1)} c^{e} \geq s_{1}^{0}
$$

${ }^{15}$ Note that the condition $\frac{\Phi(2)}{\Phi(1)}<\frac{\mathbf{E}\left(c \mid c>s_{1}^{0}\right)}{\mathbf{E}(c)}$ is the same ensuring $\zeta_{1}\left(\mathbf{s}^{1}\right)>\zeta_{0}\left(\mathbf{s}^{0}\right)$ under flexible planning. 
For agents that correctly assess the cutoff value at date $1, s_{1}^{0}=s_{1}^{1}=\Phi(1) c^{e}$, the sufficient condition (21) becomes

$$
\Phi(2) \geq \Phi(1)^{2}
$$

which holds for both exponential discounters (where $\Phi(1)=\delta$ and $\Phi(2)=\delta^{2}$ ) and sophisticated quasi-hyperbolic discounters (where $\Phi(1)=\beta \delta$ and $\Phi(2)=\beta \delta^{2}$, with $\beta \in(0,1)$ ).

For naïve quasi-hyperbolic discounters, $\Phi(1)=\beta \delta$ and $\Phi(2)=\beta \delta^{2}$, as for the sophisticated agent, but the cutoff value is $s_{1}^{0}=\delta c^{e}$ (i.e. she believes she will use the same cutoff value as an exponential discounter). Also in such a case, the sufficient condition 200 holds.

Finally, note that a sufficient condition for 20 to be negative is

$$
\Phi(2) c^{e}-\Phi(1) \underline{\mathrm{c}} \leq 0,
$$

which means that, from the perspective of date 0 , completing the task at date 1 at the lowest possible cost is worse than completing the task at date 2 .

Let $\mathbf{E}\left[c_{1}\right] \neq \mathbf{E}\left[c_{2}\right]$. Consider the following weight function: $\Phi(1)=\beta \delta$ and $\Phi(2)=$ $\beta \delta^{2}$, where $\delta, \beta \in[0,1]$. When $\beta=1$, this function represents the discounting function of an exponential discounter, while for $\beta<1$ the function is a quasi-hyperbolic discounting function. Consider the case where $\delta=1 / 2$ and $\beta=1 / 2$, and suppose the agent knows with certainty that the expected cost of completing the task at date 1 and 2 is $c_{1}=\mathbf{E}\left[c_{1}\right]=8$ and $c_{2}=\mathbf{E}\left[c_{2}\right]=24$. From the perspective of date 1, postponing task completion to date 2 is preferred to completing immediately if

$$
c_{1}>\Phi(1) \mathbf{E}\left[c_{2}\right]
$$

which clearly holds because $8>24 / 4=6$. From date 0 , however, this is not desirable because

$$
\Phi(1) \mathbf{E}\left[c_{1}\right]<\Phi(2) \mathbf{E}\left[c_{2}\right]
$$

i.e. because the expected cost of listening at date 1 , which is $8 / 4=2$, is lower than the expected cost of listening at date 2 , which is $24 / 8=3$. In other words, given this distribution of task costs, at date 1 there would be a dynamic choice reversal that is not desirable from the perspective of date 0 (see 6). Hence flexibility is not desirable and precommiting to the rigid plan assessed at date 0 is the best option. This conclusion, however, depends on the expectations on future task costs. As an example, consider the case where the cost of 
completing the task at date 1 is $c_{1}=5$. In such a case self-control problems would not arise, despite the preferences being present-biased, because it is optimal to complete the task both at date 1 and 2 (see 5).

\section{A5. A naïve quasi-hyperbolic discounter values flexibility more than a so-} phisticated quasi-hyperbolic discounter

We introduce two different notations for the cutoff value $s_{0}^{1}$ used by naïve and sophisticated agents. Let $s^{\text {naive }}=\delta c^{e}$ be the cutoff value for the naïve agent, and let $s^{s o p h}=\beta \delta c^{e}=\beta s^{n}$ be the (correct) cutoff value for the sophisticated agent (i.e. $s_{1}^{0}=s_{1}^{1}$ ). Consider treatment 1 (for treatment 2 the same logic holds). Replacing $c^{e}=s^{\text {naive }} / \delta$, the difference between the value of flexibility for a naïve and the value for a sophisticated (see 15 and 16 is as follows:

$$
\begin{aligned}
M\left(s^{\text {naive }}\right)-M\left(s^{\text {soph }}\right) & =\frac{(2 p-1)}{D(2)} \beta \delta\left[F\left(s^{n}\right)\left(s^{n}-\mathbf{E}\left[c_{1} \mid c_{1}<s^{n}\right]\right)-F\left(\beta s^{n}\right)\left(s^{n}-\mathbf{E}\left[c_{1} \mid c_{1}<\beta s^{n}\right]\right)\right] \\
& =\frac{(2 p-1)}{D(2)} \beta \delta\left[\left(F\left(s^{n}\right)-F\left(\beta s^{n}\right)\right) s^{n}-\left(\int_{\underline{c}}^{s^{n}} x f(x) d x-\int_{\underline{c}}^{\beta s^{n}} x f(x) d x\right)\right] \\
& =\frac{(2 p-1)}{D(2)} \beta \delta\left(\int_{\beta s^{n}}^{s^{n}} s^{n} f(x) d x-\int_{\beta s^{n}}^{s^{n}} x f(x) d x\right) \\
& =\frac{(2 p-1)}{D(2)} \beta \delta\left(\int_{\beta s^{n}}^{s^{n}}\left(s^{n}-x\right) f(x) d x\right) .
\end{aligned}
$$

Given that $\int_{\beta s^{n}}^{s^{n}}\left(s^{n}-x\right) f(x) d x$ is strictly positive because $x \in\left[\beta s^{n}, s^{n}\right]$, we conclude that $M\left(s^{\text {naive }}\right)>M\left(s^{\text {soph }}\right)$, which implies that a naïve quasi-hyperbolic discounter is willing to pay more for flexibility with respect to a sophisticated quasi-hyperbolic discounter. 


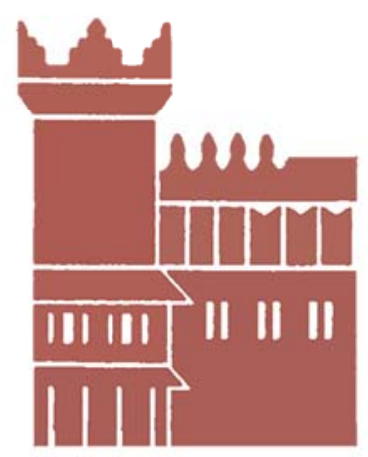

Alma Mater Studiorum - Università di Bologna DEPARTMENT OF ECONOMICS

Strada Maggiore 45

40125 Bologna - Italy

Tel. +39051 2092604

Fax +390512092664

http://www.dse.unibo.it 